\title{
Known allergen structures predict Schistosoma mansoni IgE-binding antigens in human infection
}

\section{Edward J. Farnell ${ }^{1}{ }^{*}$, Nidhi Tyagi ${ }^{2}$, Stephanie Ryan ${ }^{3}$, lain W. Chalmers ${ }^{4}$, Angela Pinot de Moira ${ }^{1}$, Frances M. Jones ${ }^{1}$, Jakub Wawrzyniak ${ }^{1}$, Colin M. Fitzsimmons ${ }^{1}$, Edridah M. Tukahebwa ${ }^{5}$, Nicholas Furnham ${ }^{2,6}$, Rick M. Maizels ${ }^{3}$ and David W. Dunne ${ }^{1}$}

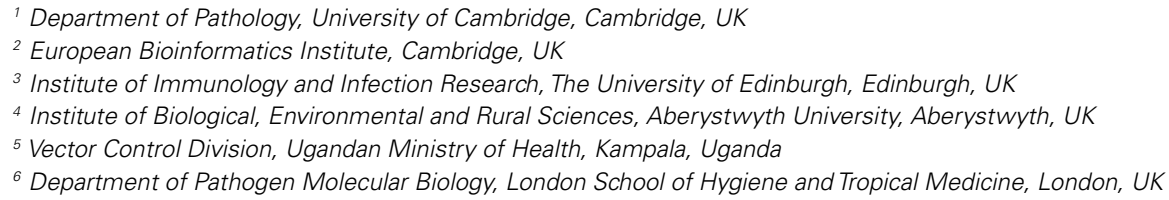

\section{Edited by:}

Abhay Satoskar, The Ohio State

University, USA

\section{Reviewed by:}

Adam Cunningham, University of Birmingham, UK

Luis I. Terrazas, Facultad de Estudios Superiores Iztacala, Mexico

${ }^{*}$ Correspondence:

Edward J. Farnell, Department of Pathology, University of Cambridge, Tennis Court Road, Cambridge CB2 1QE, UK

e-mail:ef242@cam.ac.uk
The $\lg E$ response has been associated with both allergic reactions and immunity to metazoan parasites. Recently, we hypothesized that all environmental allergens bear structural homology to IgE-binding antigens from metazoan parasites and that this homology defines the relatively small number of protein families containing allergenic targets. In this study, known allergen structures (Pfam domains) from major environmental allergen families were used to predict allergen-like (SmProfilin, SmVAL-6, SmLipocalin, SmHSP20, Sm triosephosphate isomerase, SmThioredoxin, Sm superoxide dismutase, SmCyclophilin, and Sm phosphoglycerate kinase) and non-allergen-like [Sm dynein light chain (SmDLC), SmAldolase SmAK, SmUbiquitin, and Sm14-3-3] proteins in Schistosoma mansoni. Recombinant antigens were produced in Escherichia coli and $\operatorname{lgG} 1, \operatorname{lgG} 4$, and $\operatorname{lgE}$ responses against them measured in a cohort of people $(n=222)$ infected with $S$. mansoni. All allergen-like antigens were targeted by $\operatorname{lgE}$ responses in infected subjects, whilst IgE responses to the nonallergen-like antigens, SmAK, SmUbiquitin, and Sm14-3-3 were essentially absent being of both low prevalence and magnitude. Two new IgE-binding Pfam domain families, not previously described in allergen family databases, were also found, with prevalent lgE responses against SmDLC (PF01221) and SmAldolase (PF00274). Finally, it was demonstrated that immunoregulatory serological processes typically associated with allergens also occurred in responses to allergen-like proteins in $S$. mansoni infections, including the production of lgG4 in people responding with IgE and the down-regulation of IgE in response to increased antigen exposure from $S$. mansoni eggs. This study establishes that structures of known allergens can be used to predict IgE responses against homologous parasite allergen-like molecules (parallergens) and that serological responses with IgE/lgG4 to parallergens mirror those seen against allergens, supporting our hypothesis that allergenicity is rooted in expression of certain protein domain families in metazoan parasites.

Keywords: schistosomiasis mansoni, allergenicity, $\lg \mathrm{E}$, IgG4, parasite allergens, metazoan parasite, helminth proteins

\section{INTRODUCTION}

The role of IgE in allergic immune responses is well established and extensive research has characterized $\approx 3000$ known allergens $(1,2)$. Further analysis of known allergens has shown that they occupy a highly restricted set of 255 protein domains, defined in the Pfam database by their structure and function, representing $<2 \%$ of all known protein domain families (3). Furthermore just 10 Pfam protein domains represent no fewer than $40 \%$ of the 995 molecular protein allergens described in the Allfam allergen family database. These "top 10" families containing the greatest number of different individual allergenic proteins are (in order of frequency) Prolamin, EF-hand, Tropomyosin, CAP (CRISP/Antigen-5/PR-1),
Subtilisin-like serine proteases, Lipocalin, Profilin, Bet v 1, Expansin, and Cupin (3). The reasons for the predominance of a restricted molecular subset and the principles underlying "What makes an allergen an allergen?" are currently not known. Many different biochemical properties of proteins have been suggested to be determinants of allergenicity including size, solubility, hydrophobicity, protein fold stability, oligermerization, venom toxicity, post-translational modifications, and protease activity $(2,4)$. However, none of these factors independently describe all allergens or indeed differentiate satisfactorily between allergens and non-allergens, for example, not all allergens are proteases and there are many examples of non-allergenic proteases. 
We have therefore suggested a new paradigm, which defines a common characteristic of potentially allergenic structural protein families, and explains why they are restricted in number (5). Potent IgE-mediated immune responses are seen during infections with metazoan parasites, including both helminth (worm) endoparasites and ectoparasites such as arthropod ticks. Moreover, there is a growing body of evidence to suggest that the IgE-mediated immune response, which is a recently evolved immune mechanism found only in mammals, helps protect against infection with metazoan parasites (6-13). Furthermore, given that metazoan parasites have similar cell biology to the metazoan host, it would be expected that potential targets for antibody responses would exclude proteins with high identity to their equivalents in the host. This appears to be more marked with IgE than other antibody isotypes and most environmental allergens share $<70 \%$ identity with their human equivalents (14).

Examination of the "top 10" allergen families in the context of the human antibody responses against infecting metazoan parasites revealed examples of parasite proteins with known IgEbinding domains or "parallergens" in three allergen families; EFhand domains in Tegumental-Allergen-Like (TAL) proteins from schistosomes (15), Tropomyosin proteins in filarial nematodes and schistosomes $(16,17)$, and CAP protein domains in hookworm nematodes (18). A further five families; Subtilisin-like serine protease, Lipocalin, Profilin, Bet v 1, and Cupin families, contain metazoan parasite homologs with the potential to be targeted by IgE during human parasitic infections (5). Additionally there are many known immunogenic proteins, with potential IgE-binding activity, within the most abundant schistosome proteins that may have homology to known allergens (19-22).

When attempting to identify IgE responses to parasite proteins it is important to consider the effects of chronic helminth infection in humans, and the shifts between IgE and IgG4 isotypes that occur during long-term exposure (23). Responses to parallergens in helminth-infected subjects may result in the down-regulation of IgE antibodies and the production of IgG4 to the same allergenic epitopes $(15,24)$. This shift is well-documented in allergy, especially in specific immunotherapy (SIT), where constant lowlevel exposure to a specific allergen results in increased IgG4 and decreased IgE levels $(25,26)$. In individuals with schistosomiasis mansoni, the expression of proteins across the S. mansoni life cycle and their localization in specific tissues may be important in determining the levels of exposure of a protein to the immune system and the corresponding antibody responses. The human life cycle stages of S. mansoni include infective skin-penetrating cercaria, host tissue-migrating schistosomule, blood-dwelling adult worms, and eggs (27). Due to a number of immune evasion mechanisms, protein antigens in cercaria, schistosomules, and adult worms are generally sequestered away from immune system and are only exposed upon the death of the adult worms, which may take between 3 and 10 years (28). On the other hand, antigens present in eggs (thousands of which are released daily) are continuously exposed to the immune system during the death of eggs that have failed to escape the host and become trapped in tissues, a process, which usually takes 1-2 weeks. Indeed studies in schistosomiasis mansoni endemic areas on the SmTAL family of EF-hand parallergens have shown that for antigens expressed exclusively in the adult worm stage of the parasite, such as SmTAL1 and SmTAL3, IgE responses only develop naturally over time as adult worms die or during treatment with praziquantel (PZQ), which damages adult worms and reveals cryptic antigens (24). Conversely, for antigens expressed strongly in eggs, such as SmTAL2, IgE antibodies are seen only during early infection (children $<5$ years) with IgE responses down regulated and replaced with $\operatorname{IgG} 4$ antibody responses in older individuals $(24,29)$. Therefore in determining the "allergen-like" properties of S. mansoni predicted parallergens it is important to consider their expression in eggs/adult worms, as well as both IgE and IgG4 antibody levels to each antigen.

We therefore hypothesize that known IgE-binding domains may be used to predict the protein targets of allergy-like responses in individuals infected with $S$. mansoni, including targeting by IgE, the induction of regulatory responses such as targeting by IgG4 and the down-regulation of IgE in response to continuous exposure of antigens during infection. In this study, we now show that known allergen structures can indeed be used to predict IgEbinding and allergy-like responses against recombinant $S$. mansoni parallergens in an infected human cohort from a schistosomiasis mansoni endemic area of Uganda.

\section{MATERIALS AND METHODS PARASITE MATERIAL}

A Puerto Rican strain of S. mansoni was used in this study. RNA was isolated and extracted from cercaria and adult worms as previously described (30). RNA integrity was verified using a Bioanalyser (Agilent Technologies, Bracknell, UK). cDNA was prepared from $1 \mu \mathrm{g}$ total RNA using random hexamers (SigmaAldrich, Gillingham, UK) and Superscript II reverse transcriptase according to the manufacturer's instructions (Life Technologies, Paisley, UK).

\section{BIOINFORMATIC ANALYSIS}

Hidden Markov Models (HMMs) of Pfam domains associated with the "top 10" allergen families listed in Allfam ${ }^{1}$ were used to search the Uniprot database ${ }^{2}$ to identify any S. mansoni proteins containing the relevant Pfam domains $(3,31)$. Similarly Pfam domains associated with $S$. mansoni proteins abundant in human life cycle stages [as identified by proteomics (21)] were used to search Allfam for functional sequence similarity to families of known allergens.

To compare known parasite and allergen structures, protein sequences of $S$. mansoni proteins with known 3D structure were used to search Allergome ${ }^{3}$ for allergens with similar sequence. Allergens with an $E$-value cut-off of $<1 \times 10^{-4}$ were searched for corresponding known 3D structures in Protein Data Bank ${ }^{4}$. Protein models were generated using MODELLER v9.10 (32) and LSQMAN $^{5}$ was used to perform protein structural alignments by least squares super-positioning with the quality of the models assessed by root mean squared deviation (RMSD).

\footnotetext{
${ }^{1}$ www.meduniwien.ac.at/allergens/allfam/

${ }^{2} \mathrm{http}: / /$ www.uniprot.org

${ }^{3}$ http://www.allergome.org/

${ }^{4}$ http://www.ebi.ac.uk/pdbe/

${ }^{5}$ http://xray.bmc.uu.se/usf/lsqman_man.html
} 


\section{MOLECULAR CLONING}

SmTAL1 (GenBank M37003.1), SmTAL2 (GenBank M67506.1), Sm triosephosphate isomerase (SmTPI) (GenBank M83294.1), and SmTropomyosin II (GenBank KC904504.1) were produced using the pGEX family of plasmids during other studies (17, 24, 33). pGEX-KG plasmid containing Sm dynein light chain (SmDLC) (GenBank U55992.1) was a kind gift from Karl Hoffmann (University of Aberystwyth). The SmVAL-6 CAP domain (amino acids 1-145 of full-length SmVAL-6, GenBank AY953433) was cloned into the pET-30a vector. All other antigens were cloned in the pGEX-KG vector. Gene sequences were derived from GenBank and primers containing unique restriction enzymes sites were designed using Primer3Plus (Table S1 in Supplementary Material $)^{6}$. PCR to generate gene specific coding sequences was performed using Phusion High Fidelity DNA polymerase (Thermo Fisher Scientific, Reading, UK) in a Tetrad 2 thermal cycler (MJ research/Bio-Rad, Hemel Hempstead, UK). PCR products and plasmids were digested with the relevant pair of FastDigest restriction enzymes (Table S1 in Supplementary Material). Additionally, plasmids were treated with FastAP (Thermo Fisher Scientific) to prevent self-ligation and ligation was performed using T4 DNA ligase (Thermo Fisher Scientific). Ligated plasmids were used to transform chemically competent $\mathrm{DH} 5 \alpha$ E. coli cells by heat shock. Sanger sequencing was used to characterize cloned sequences. The sequences of SmLipocalin, Sm phosphoglycerate kinase (SmPGK), SmCyclophilin, and SmAldolase were found to be identical to previously cloned and sequenced cDNAs; GenBank Accession numbers M60895.1, L36833.1, L46884.1, and L38658.1. For molecules that had not been previously cloned and sequenced or differed from previous submissions, sequences were uploaded to GenBank with the following accession numbers; SmProfilin, KJ545485; Sm14-3-3, KM281669; SmUbiquitin, KM281670; SmAK, KM281671; SmThioredoxin, KM281672; Sm superoxide dismutase (SmSOD), KM281673; and SmHsp20, KM281674.

\section{PROTEIN EXPRESSION AND PURIFICATION}

SmTAL1, SmTAL2, SmTPI, and SmTropomyosin II were expressed and purified as described previously $(17,24,33)$. All other proteins ligated into pGEX-KG were expressed and purified as follows. Overnight cultures of TG2 E. coli containing the relevant plasmid were diluted 1:10, expanded to OD 0.4-0.8 and induced for $3-5 \mathrm{~h}$ with $1 \mathrm{mM}$ IPTG. For SmSOD, TG2 E. coli cultures were also supplemented with $0.02 \mathrm{mM} \mathrm{CuCl}_{2}$ and $0.02 \mathrm{mM} \mathrm{ZnCl}_{2}$ and induced with 0.1 M IPTG as defined by Cardoso et al. (34). Cells were lysed using a French Pressure Cell at $\approx 10,000$ psi and GST-fusion proteins isolated by affinity chromatography on Glutathione Sepharose 4B using an AKTAprime plus (GE Healthcare, Amersham, UK) as per the manufacturer's instructions. Proteins were separated from their fusion partners by cleavage on Glutathione Sepharose using thrombin (GE Healthcare). Contaminating GST-fusion partner and thrombin were removed by incubation with Glutathione Sepharose 4B (GE Healthcare) and benzamadine-agarose beads (Sigma-Aldrich), respectively.

${ }^{6}$ http://primer3plus.com
SmVAL-6 was purified using a combination NiNTA and QSepharose affinity chromatography as follows. Overnight cultures of BL21(DE3) E. coli containing the SmVAL-6 construct were cultured, induced, and lysed as above. Hexahistidine tagged proteins were isolated by affinity chromatography on NiNTA-agarose using an AKTAprime plus (GE Healthcare) as per the manufacturer's instructions. Bacterial protein contamination was removed by QSepharose ion exchange using a HiTrap Q (FF) column. Proteins were separated by elution on a $0-1 \mathrm{M} \mathrm{NaCl}$ gradient, and fractions containing only SmVAL-6 (120-350 mM NaCl) reserved. Proteins were tested for GST and bacterial contamination by in-house enzyme-linked immunosorbent assay (ELISA) assay using rabbit anti-GST antisera (Sigma) and rabbit anti-E. coli lysate antisera as previously described (24). Where required, MALDI fingerprinting was performed by the Protein and Nucleic Acid Chemistry facility (University of Cambridge Department of Biochemistry, Cambridge, UK).

\section{STUDY POPULATION}

Venous blood samples were collected from volunteers living in the fishing village of Namoni, on the shores of Lake Victoria in the Mayuge district of South East Uganda. Transmission of schistosomiasis was consistently high throughout the year and the community received annual treatment as part of the Ugandan national control program. Three hundred seventy-two individuals (6-40 years, 57\% female, mean age 18) with detectable infection (based on one stool sample, two Kato-Katz thick smears) were randomly selected from the village. Blood samples were collected, immediately prior to chemotherapy with PZQ $(40 \mathrm{mg} / \mathrm{kg}$ body mass) and 5 weeks post-treatment, into heparinized tubes. Plasma was isolated by centrifugation at $2000 \times g$ for $5 \mathrm{~min}$, then aliquoted and stored at $-80^{\circ} \mathrm{C}$ until use. Parasitological examinations were carried out before treatment and at 5 weeks post-treatment (to determine efficacy of treatment). For this study, we focused on 222 individuals ( $60 \%$ of total) for whom full serological and parasitological data were available (age 6-40 years, mean age 16 years, $59 \%$ female, pre-treatment prevalence of $S$. mansoni infection was $98.2 \%)$.

\section{ETHICS STATEMENT}

The Ugandan National Council of Science and Technology provided ethical clearance. Forms were translated in the local language and informed written assent was obtained from all adults and written consent from the parents/legal guardians of all children under 15 .

\section{ENZYME-LINKED IMMUNOSORBENT ASSAY}

Antigen-specific IgG1, IgG4, and IgE levels were measured in the plasma of infected individuals in duplicate by ELISA as described previously (24). The following modifications were made for specific antigens used in this protocol. Each antigen was applied to 384 well plates and incubated overnight at $4^{\circ} \mathrm{C}$ or dried to the plates at $37^{\circ} \mathrm{C}$ overnight as indicated in Table S2 in Supplementary Material. For each antigen, saturation coating concentrations and conditions were determined using an in-house competitive binding ELISA and were as shown in Table S2 in Supplementary Material. Human plasma was diluted 1:40 for IgE assays and 1:100 for IgG isotypes. 


\section{STATISTICAL ANALYSIS}

Data management and statistical analysis was performed using STATA for Mac version 12.1. Change in the magnitude of antibody levels following treatment with PZQ were calculated as follows:

$\%$ change $=100$ $\times\left[e^{\bar{x}(\ln (\text { AbMagnitudepost }-\mathrm{RX}+0.01)-\ln (\text { AbMagnitudepre }-\mathrm{RX}+0.01))}-1\right]$

Significance in the changes of magnitudes were calculated by Wilcoxon matched-pairs signed-ranks test. Prevalences/proportions of responders were calculated following the application of assay sensitivity cut-offs $($ mean $+3 \sigma)$ determined using sera from 26 normal European controls. Final cut-off values were determined following correction for outliers (NES responses greater than mean $+3 \sigma$ ) using in iterative process in which outliers were removed and the threshold recalculated until no outliers were found (Table S3 in Supplementary Material). Comparison of proportions of individuals responding with IgE alone vs. those responding with IgE and IgG4 together was performed by twosample test of proportions. Significance in the difference in of antigen expression levels by microarray was calculated by one way ANOVA with Bonferroni post-tests for antigen-to-antigen differences. Correlations between IgE and egg expression levels were calculated using linear regression followed by Cook's distance and leverage-vs.-squared-residual plot regression diagnostics to assess the impact of influential outliers. Regression diagnostics were performed to determine that assumptions were valid including, linearity, normal distribution of errors, and homoscedasticity of variance.

\section{RESULTS}

\section{IDENTIFICATION OF ALLERGEN-LIKE ANTIGENS IN S. MANSONI}

Protein domain searches were used to identify S. mansoni proteins with functional domain sequence similarity (similar Pfam domains) to known allergens. Firstly, we searched for examples of S. mansoni proteins with Pfam domain similarity to allergens from the "top 10" Allfam allergen families. Proteins with similarity to the EF-Hand, Profilin, CAP, Tropomyosin, Lipocalin, and Subtilisin-like Serine Protease families listed in Allfam were found in S. mansoni (Table 1), whilst examples of proteins in the Bet v 1, Cupin, Expansin, and Prolamin families were not. Secondly, we searched for potential parallergens amongst the $32 \mathrm{~S}$. mansoni proteins that were identified in the proteomic study of
Curwen et al. (21) as being abundant in lifecycle stages found in the human host. Twenty-four (75\%) were found to have domains with sequence similarity to known allergens (Table 2) whilst 8 (25\%) did not.

From these two approaches, nine predicted parallergens were selected for study, SmProfilin, SmVAL-6, SmLipocalin, SmThioredoxin, SmCyclophilin, SmTPI, SmSOD, SmPGK, and SmHSP20. Additionally, 5 S. mansoni proteins were selected that were abundant within the parasite, but were not predicted to be parallergens, including SmAldolase, SmUbiquitin, SmDLC, SmAK, and Sm14-3-3. Finally, known IgE target proteins, SmTAL1, SmTAL2, and SmTropomyosin II produced for other studies were used as positive controls $(17,24)$.

\section{CLONING AND EXPRESSION OF ANTIGENS IN E. COLI}

Recombinant forms of the antigens selected for cloning were produced in E. coli. In the case of SmVAL-6 only the soluble invariant $\mathrm{N}$-terminal segment of the protein (amino acids 1-145) containing the CAP domain was expressed. For each protein, coding sequences were cloned from parasite cDNA by PCR. These were inserted into either the pGEX-KG or pET$30 \mathrm{a}$ vector and sequenced. Following expression and extensive purification molecular weights of the recombinant proteins were estimated by SDS-PAGE (Figure 1); SmVAL-6, $19 \mathrm{kDa}$; SmLipocalin, $15.5 \mathrm{kDa}$; SmProfilin, $15 \mathrm{kDa}$; Sm14-3-3, $26 \mathrm{kDa}$; SmDLC, $12 \mathrm{kDa}$; SmCyclophilin, $20 \mathrm{kDa}$; SmAldolase, 41 kDa; SmPGK, 45 kDa; SmHSP20, 42 kDa; SmUbiquitin, 16 kDa; SmSOD $17 \mathrm{kDa}$; SmThioredoxin $10 \mathrm{kDa}$; SmTPI $27 \mathrm{kDa}$; and SmAK $24 \mathrm{kDa}$. In the Sm14-3-3 sample, proteins observed at approximately 18 and $8 \mathrm{kDa}$ represent the $\mathrm{C}$ and $\mathrm{N}$-terminal (respectively) degradation products of full-length Sm14-3-3 as confirmed by MALDI fingerprinting (data not shown). Assay by ELISA confirmed that all recombinant proteins were free from significant contamination from the GST-fusion partner and bacterial antigens (data not shown).

\section{IgE RESPONSES ARE PREVALENT AGAINST PREDICTED ALLERGEN-LIKE MOLECULES}

In order to determine the immunogenicity of the antigens, serological responses were studied in detail. For each antigen, three antibody isotypes, IgE, IgG4, and IgG1, were measured in plasma from an infected cohort living in a schistosomiasis mansoni endemic area of Uganda. Previous studies have indicated that some antigens may be sequestered within the adult worms and not exposed

Table 1 | Schistosoma mansoni antigens with functional sequence identity to abundant allergens.

\begin{tabular}{lclll}
\hline Protein & Pfam domain & Pfam name & Allergen family name (Allfam) \\
\hline SmTAL1 & PF00036 & EF-Hand & EF-hand domain & UniProt identifier \\
SmProfilin & PF00235 & Profilin & Profilin & Q4TTW6 \\
SmVAL-6 & PF00188 & Cysteine-rich secretory protein family & CRISP/PR-1/venom group 5 allergen family \\
SmTropomysosin II & PF00261 & Tropomyosin & Tropomyosin & G4V6N1 \\
SmLipocalin & PF00061 & Lipocalin/cytosolic fatty-acid binding & Lipocalin & \\
& & protein family & G4VN65 \\
SmFurin-1 & PF00082 & Subtilase family & Subtilisin-like serine protease
\end{tabular}


Table 2 | Identification of allergen-like proteins in abundant proteins from S. mansoni as identified in proteomic studies by Curwen et al. (21).

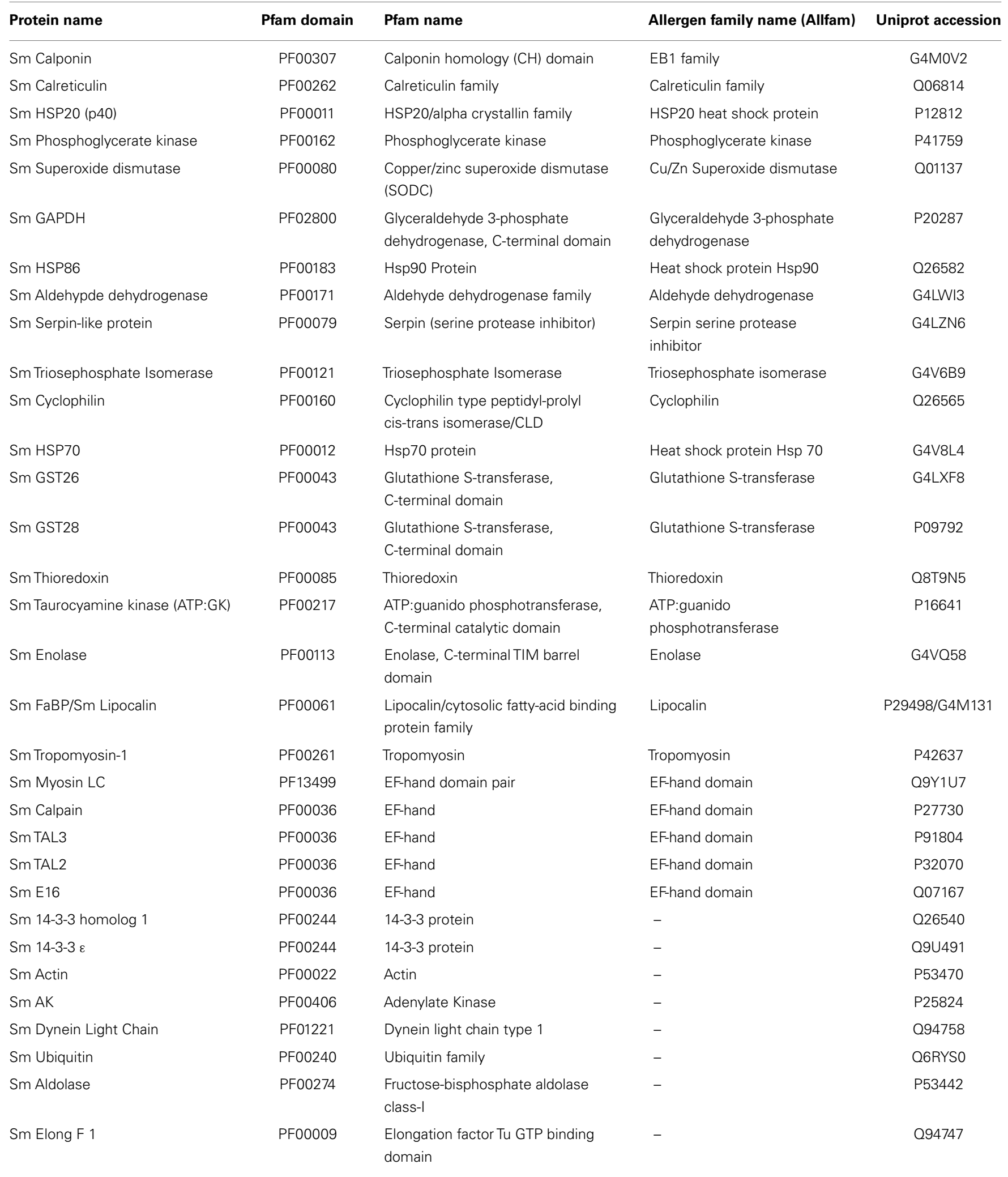

For proteins with functional similarity to known allergens the Allfam allergen family name is given. 
to the immune system (24). Therefore antibody levels were studied 5 weeks post-treatment with PZQ so as to observe immune responses that may only be generated following worm damage or death. It is recognized that the use of a single dilution of sera per antibody isotype is a limitation of this study but it was a necessity given the number of individuals and sera isotypes assayed.

All nine of the predicted parallergens including SmHSP20, SmVAL-6, SmProfilin, SmThioredoxin, SmTPI, SmCyclophilin, SmLipocalin, SmSOD, and SmPGK were found to be the target of an IgE response both pre- and post-treatment (RX) with PZQ within varying portions of the population (Figure 2A, I). The proportions ranged from 59\% post-RX for SmHSP20 to $4.1 \%$ pre-RX for SmPGK. For these antigens, the magnitudes of IgE responses ranged from $0.15 \mathrm{ng} / \mathrm{ml}$ for SmPGK pre-RX and $159.4 \mathrm{ng} / \mathrm{ml}$ for SmVAL-6 post-RX (Table 3). IgE responses were also seen against the positive control parallergens SmTAL1 and SmTropomyosin II (Figure 2A, III). Significant boosts in the magnitude of IgE responses following treatment occurred for SmProfilin, SmVAL-6, SmHSP20, and SmDLC (Table 3).

Of the five antigens that did not contain known allergenic domains, three of the antigens, SmAK, SmUbiquitin, and Sm14-33 were found to have low prevalences of IgE responders; $1.3 / 3.1 \%$, $2.7 / 0.9 \%$, and $0.9 / 0.45 \%$ pre-/post-RX, respectively (Figure 2A, II). Conversely SmDLC and SmAldolase had a relatively large proportion of specific IgE responders; $34.2 / 51.3 \%$ and $31.1 / 27.9 \%$ pre/post-RX, respectively. As shown in previous endemic study populations that did not include young children ( $<5$ years), IgE responses to SmTAL2 were absent despite its similarity to allergens from the EF-hand family $(15,24)$.

\section{IgG4 RESPONSES ARE PREVALENT AGAINST ALLERGEN-LIKE IgE-BINDING ANTIGENS}

It has been suggested that production of IgG4 against S. mansoni allergen-like proteins can be induced as part of the regulatory response to IgE-mediated inflammation and should therefore be considered as part of an allergy-like response (24). In this study, IgG4 responses were observed against all of the IgE-binding antigens studied both pre- and post-RX with PZQ (Figure 2B) with the most prevalent against SmThioredoxin $(75.2 \%$ pre-RX) and the least against SmProfilin (5.9\% pre-RX). Furthermore for the parallergens SmHSP20, SmDLC, SmThioredoxin, SmTropomyosin II, SmAldolase, SmVAL-6, and SmTAL1 the majority of individuals that produced an IgE response also produced an IgG4 response against the same antigen with a significantly greater proportion of individuals producing both IgE and IgG4 responses than an IgE response alone (Figure 3). For SmAK and Sm14-3-3 to which low prevalence IgE responses were observed, IgG4 responses were also of low prevalence. Of note was SmTAL2, which despite the absence of IgE responses was the target of specific IgG4 responses in 35 and $32 \%$ of the population pre-/post-RX, respectively. Similarly in the case of SmUbiquitin, despite a low proportion of IgE responders in the population, prevalent IgG4 responses occurred.

\section{IgG1 RESPONSES ARE PREVALENT AGAINST RECOMBINANT S. MANSONI PROTEINS}

All of the antigens, both allergen- and non-allergen-like, were found to elicit IgG1 responses in human infections both pre- and

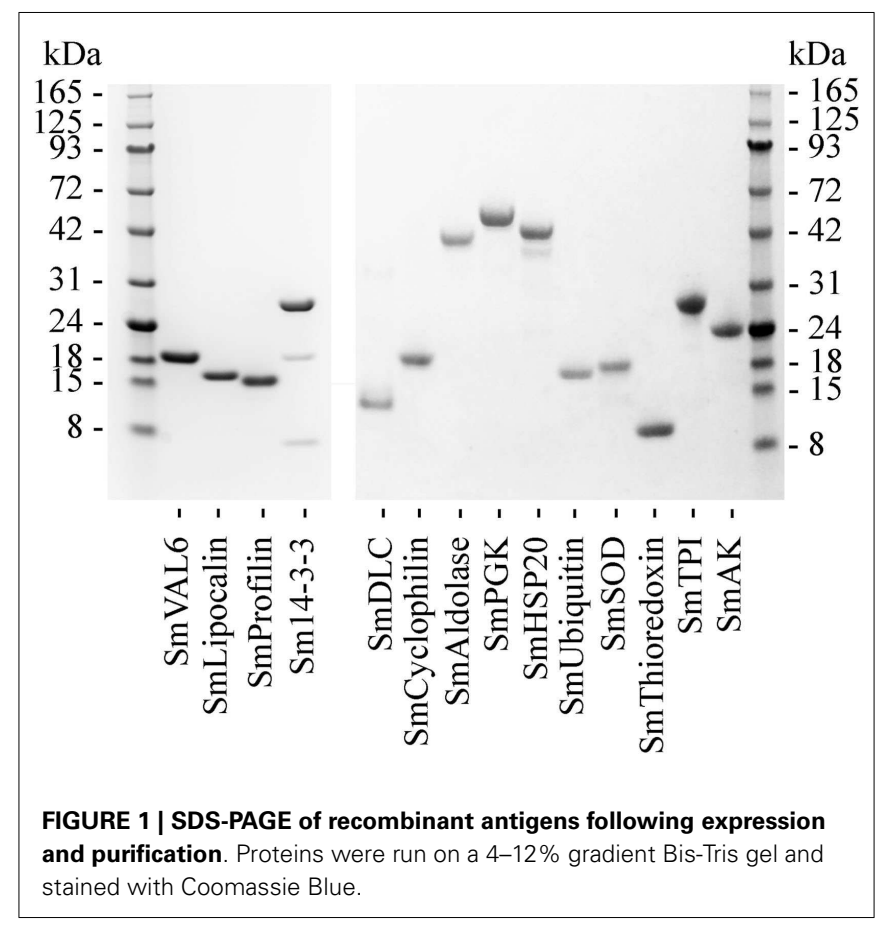

post-treatment with PZQ. IgG1 responses were found to be present against all of the recombinant antigens ranging in prevalence from $6.3 \%$ post-RX for SmTropomyosin II to $70.7 \%$ pre-RX for SmTAL2 (Figure 2C). Magnitudes of IgG1 response amongst responders were between $0.2 \mu \mathrm{g} / \mathrm{ml}$ pre-RX for SmDLC and $151.1 \mu \mathrm{g} / \mathrm{ml}$ preRX for SmTropomyosin II (Table 3). Significant differences in the magnitudes of IgG1 responses following treatment with PZQ were seen for SmTAL1, SmVAL-6, SmCyclophilin, SmThioredoxin, SmTPI, SmSOD, SmDLC, SmUbiquitin, SmAK, and Sm14-3-3 (Table 3).

\section{PARALLERGENS AND ALLERGENS HAVE CLOSE CONFORMATIONAL SIMILARITY}

Confirmed S. mansoni parallergens with previously elucidated 3D structures, SmLipocalin (1VYF), SmSOD (1TO4), and SmThioredoxin (2XBI), were compared for gross structural similarity to allergens with known 3D structure including Dermatophagoides farinae Der f 13 (2A0A), Solanum lycopersicum Superoxide dismutase (3HOG), and Litopenaeus vannamei Thioredoxin (3ZZX), respectively. All three proteins were found to have overall structural similarity with their counterpart allergens with RMSD scores of $0.9 \AA, 0.6 \AA$, and $1.5 \AA$ for SmThioredoxin/LvThioredoxin, SmSOD/SISOD, and SmLipocalin/Der f 13, respectively (Figure 4).

\section{ANTIGENS ARE DIFFERENTIALLY TRANSCRIBED IN S. MANSONI ADULT AND EGG LIFE CYCLE STAGES}

Previous studies have suggested that differing levels of S. mansoni antigen exposure may influence the prevalence and magnitude of antigen-specific antibody responses $(15,24)$. Available microarray data (35) show that all of the antigens in this study were transcribed in adult worms (Figure 5A), and whilst transcription was not equal across all antigens (SmThioredoxin and SmProfilin 


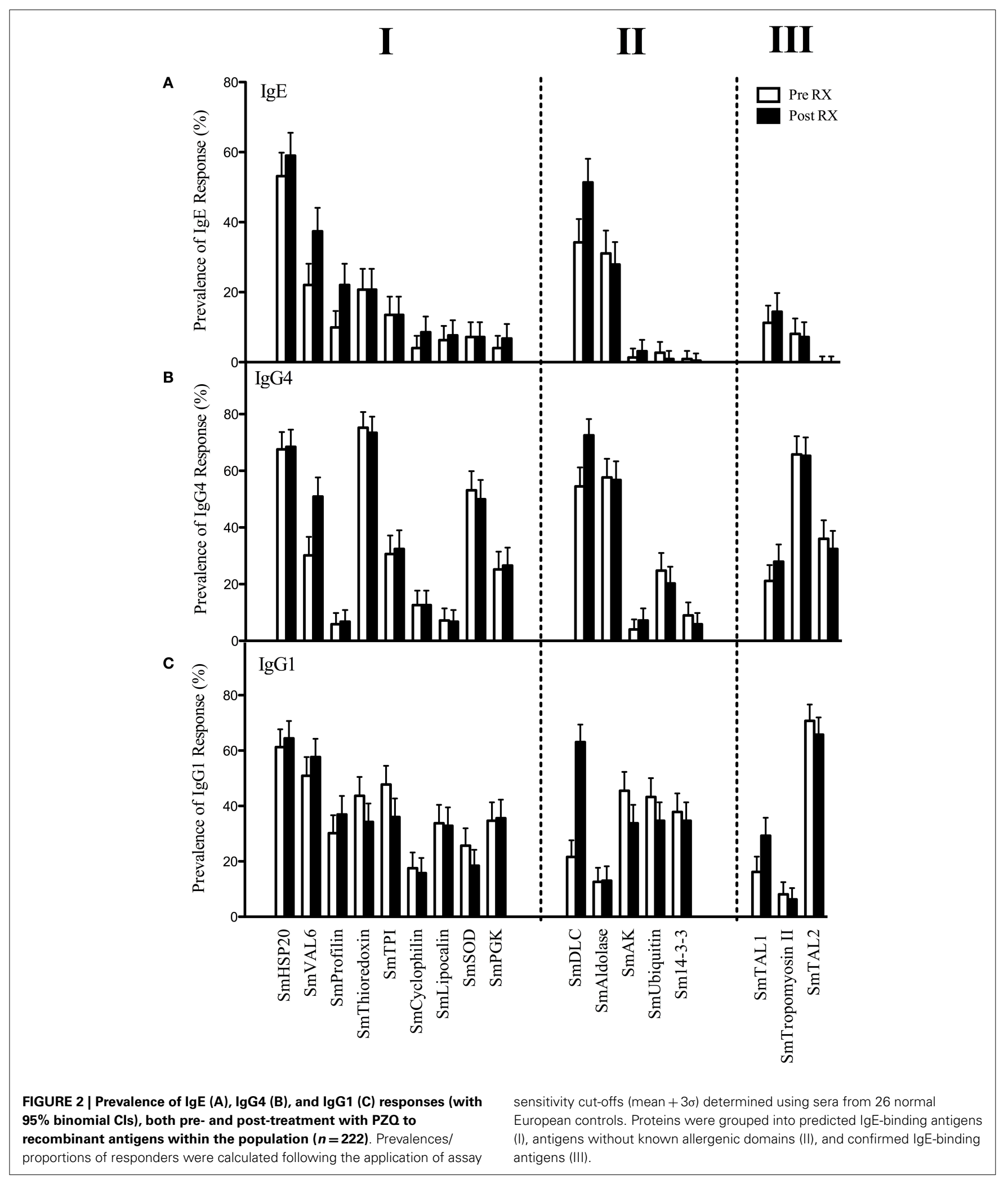

were found to be significantly lower in expression than all of the antigens) there were no antigens for which transcription in adult worms was very low or absent. Transcription of antigens in eggs (Figure 5B) was more selective with some antigens showing significantly lower expression than others (SmVAL-6, SmTAL1, SmThioredoxin, SmDLC vs. all other antigens, $p<0.001)$ and 
Table 3 | Magnitudes of antibody responses against recombinant $S$. mansoni antigens in a population of 222 infected individuals as geometric means (Ab magnitude + 0.01) of $\operatorname{lgG} 1$, IgG4, and $\operatorname{lgE}$ for sub populations of individuals with responses to $S$. mansoni antigens either pre- or post-treatment with PZO.

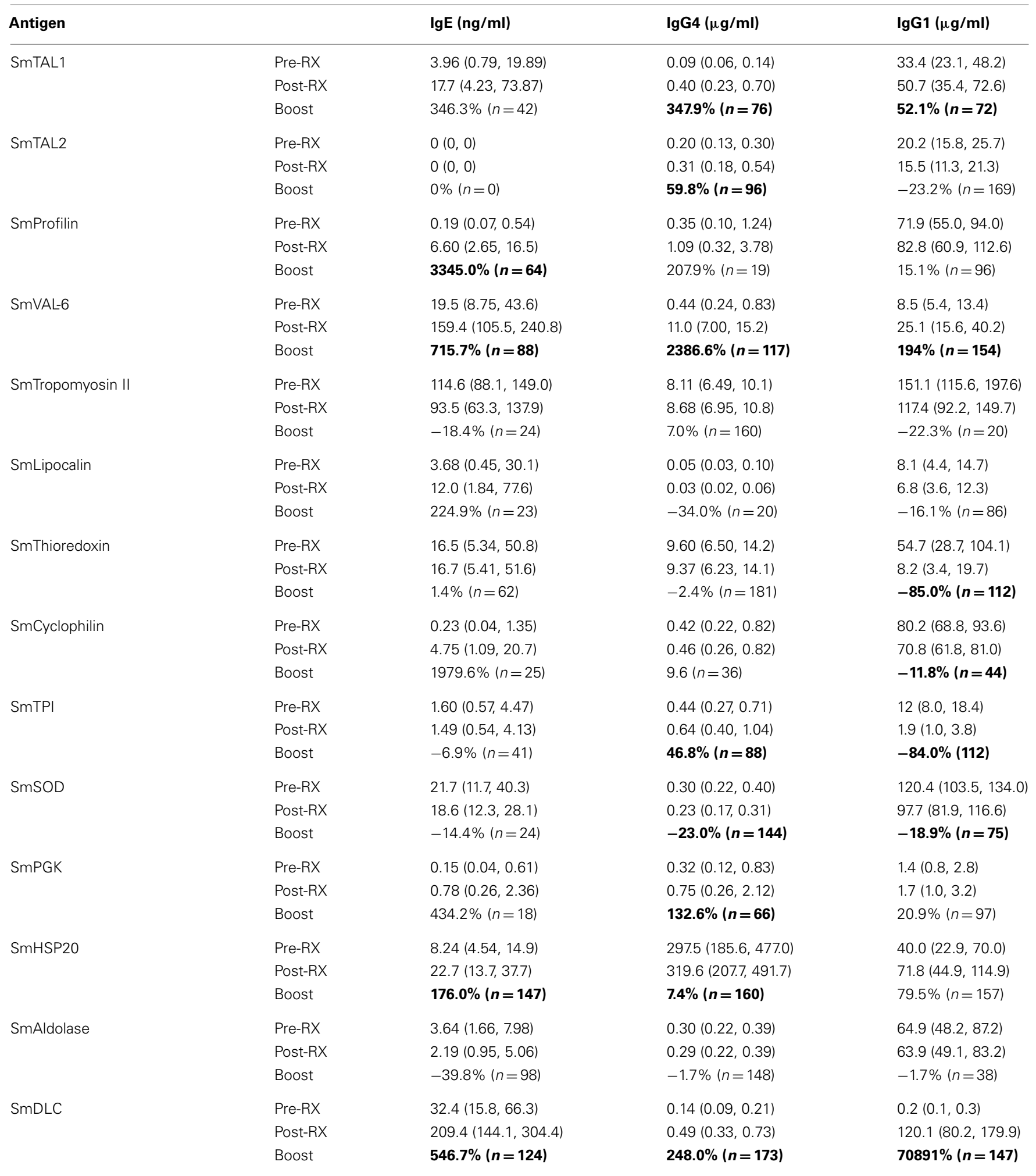


Table 3 | Continued

\begin{tabular}{|c|c|c|c|c|}
\hline Antigen & & $\lg E(n g / m l)$ & $\operatorname{lgG4}(\mu \mathrm{g} / \mathrm{ml})$ & $\operatorname{lgG1}(\mu \mathrm{g} / \mathrm{ml})$ \\
\hline \multirow[t]{2}{*}{ SmUbiquitin } & Pre-RX & $3.78(0.23,61.3)$ & $1.35(0.71,2.56)$ & $13.4(8.5,21.1)$ \\
\hline & Post-RX & $0.07(0.00,1.82)$ & $0.59(0.28,1.25)$ & $3.1(1.6,6.3)$ \\
\hline \multirow[t]{2}{*}{ SmAK } & Pre-RX & $0.13(0.01,2.61)$ & $0.08(0.02,0.28)$ & $10.6(6.5,17.1)$ \\
\hline & Post-RX & $2.93(0.22,39.1)$ & $0.22(0.10,0.49)$ & $2.0(1.0,4.0)$ \\
\hline \multirow[t]{3}{*}{ Sm14-3-3 } & Pre-RX & $2.33(0.00,309075)$ & $0.84(0.36,1.94)$ & $10.3(5.9,18.1)$ \\
\hline & Post-RX & $0.10(0.00,2569)$ & $0.21(0.06,0.72)$ & $6.1(3.1,11.9)$ \\
\hline & Boost & $-95.5 \%(n=3)$ & $-75.2 \%(n=21)$ & $-41.3 \%(n=96)$ \\
\hline
\end{tabular}

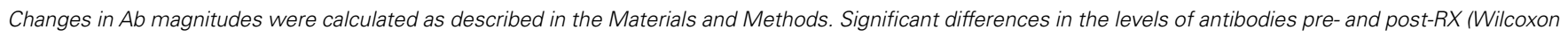
matched-pairs signed-ranks test $p<0.05)$ are highlighted in bold.

$n$, Number of responders in each group.

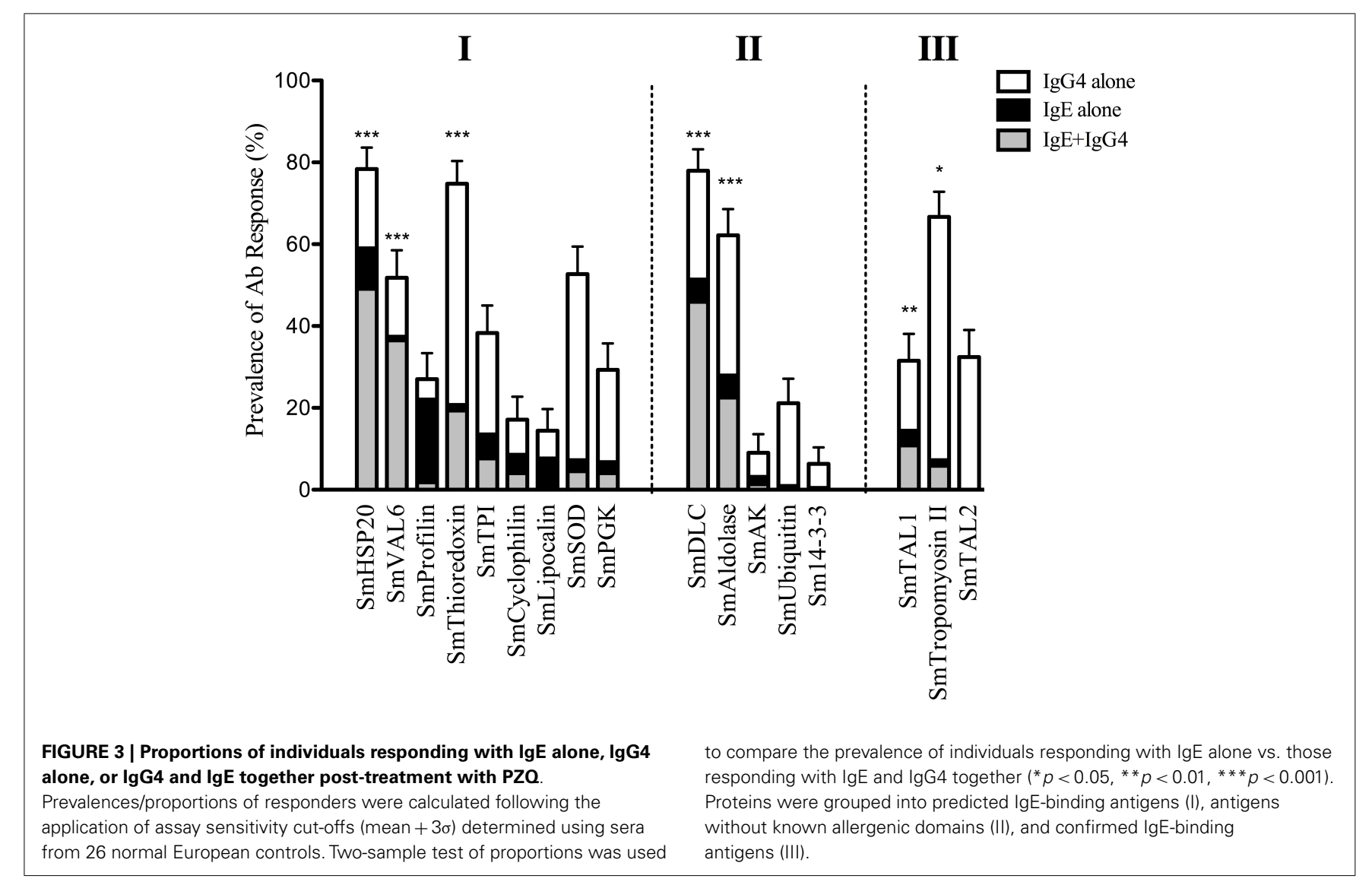

some significantly higher than others (SmHSP20 vs. all other antigens $p<0.001)$. SmVAL- 6 and SmDLC, both transcribed at lowlevels in eggs ( 0.5 and $7.7 \%$ of adult levels, respectively) showed marked boosts in antibody levels following treatment, whilst those more strongly expressed in egg, such as SmSOD, SmUbiquitin, and Sm14-3-3 (between 47.5 and $82.5 \%$ of adult transcription levels) all showed marked decreases in antibody levels post-treatment (Table 3).

\section{ANTIGEN-SPECIFIC LEVELS OF IgE ARE NEGATIVELY CORRELATED WITH ANTIGEN EXPRESSION IN EGGS}

In order to determine if constant exposure of antigens from the death of short-lived eggs in host tissues influenced levels of IgE, we compared the level of antigen expression in eggs with antigenspecific IgE levels in infected individuals both pre- and posttreatment with PZQ. As previous studies have demonstrated that the age of infected individuals, their level of IgG4 antibodies and 


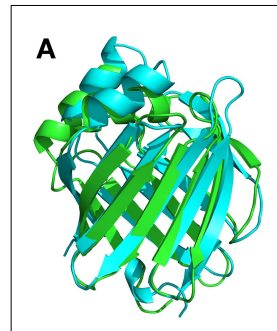

Identity: $39.39 \%(9 \mathrm{e}-25)$ RMSD: $1.5 \AA$

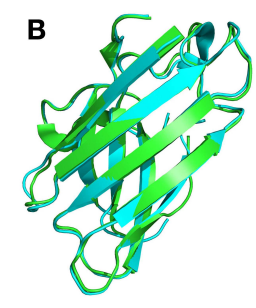

Identity: $61.74 \%(1 \mathrm{e}-49)$ RMSD: $0.6 \AA$

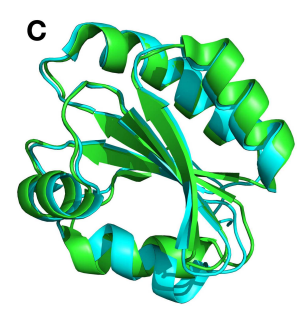

Identity: $46 \%(1 \mathrm{e}-22)$ RMSD: $0.9 \AA$

FIGURE 4 | Comparison of the structures of SmLipocalin, SmThioredoxin, SmSOD with their respective IgE-binding counterparts identified from Allergome. Parasite allergen-like molecules were used to identify similar allergens by BLAST search of the Allergome database. Identities of the matched allergens with the corresponding $E$-values are shown below each matched pair. Structures were superimposed using LSOMAN and the quality of the alignment assessed by root mean squared deviation (RMSD). (A) Alignment of SmLipocalin (1VYF, cyan) and Der f 13 (2A0A, green). (B) Alignment of SmSOD (1TO4, cyan) and Tomato SOD (3HOG, green). (C) Alignment of SmThioredoxin (2XBI, cyan) and White Shrimp Thioredoxin (3ZZX, green).

intensity of infection (as measured by number of eggs in feces) may confound the levels of IgE antibodies, these variables were included in univariate regression models explaining variation in IgE magnitude (Figure 6) (17, 29, 36-38). Regression diagnostics indicated that SmHSP20 exerted undue influence over the effect of egg transcription on IgE levels (Cook's distance $>1$ ) and was therefore excluded from this model. Upon the exclusion of SmHSP20 this analysis established that antigen-specific IgE magnitude prior to treatment was significantly negatively correlated with transcription levels for each antigen in eggs $\left(\beta=-0.550, r^{2}=0.2739\right.$, $p=0.038)$ an association that strengthened post-treatment with PZQ $\left(\beta=-0.716, r^{2}=0.3666, p=0.013\right)$ as shown in Figure 6A. None of the other confounding variables, including geometric mean (GM) age, GM infection intensity, or GM IgG4 levels were found to significantly correlate with IgE levels.

\section{DISCUSSION}

Allergy and atopic spectrum diseases have been extensively studied over a number of decades providing a detailed description of many of the protein allergens that are associated with IgE-mediated allergic immune reactions $(1,3)$. We have suggested that the immune responses associated with allergy are the by-product of the highly evolved immune response to metazoan parasites, and that allergens and metazoan IgE-binding proteins share similar structural features, which define the immune responses produced against them (5). To this end, we hypothesized that known protein allergen structures could be used to predict IgE-binding proteins from the metazoan parasite S. mansoni.

We used a bioinformatic approach to search for $S$. mansoni parallergen examples of abundant common allergens and to predict parallergens in proteins abundant in S. mansoni. Nine potential IgE-binding antigens were cloned in E. coli alongside, three known IgE targeted S. mansoni antigens, and five other abundant S. mansoni antigens that were predicted not to bind IgE due to the lack of known similar proteins in allergy. We then tested if these antigens

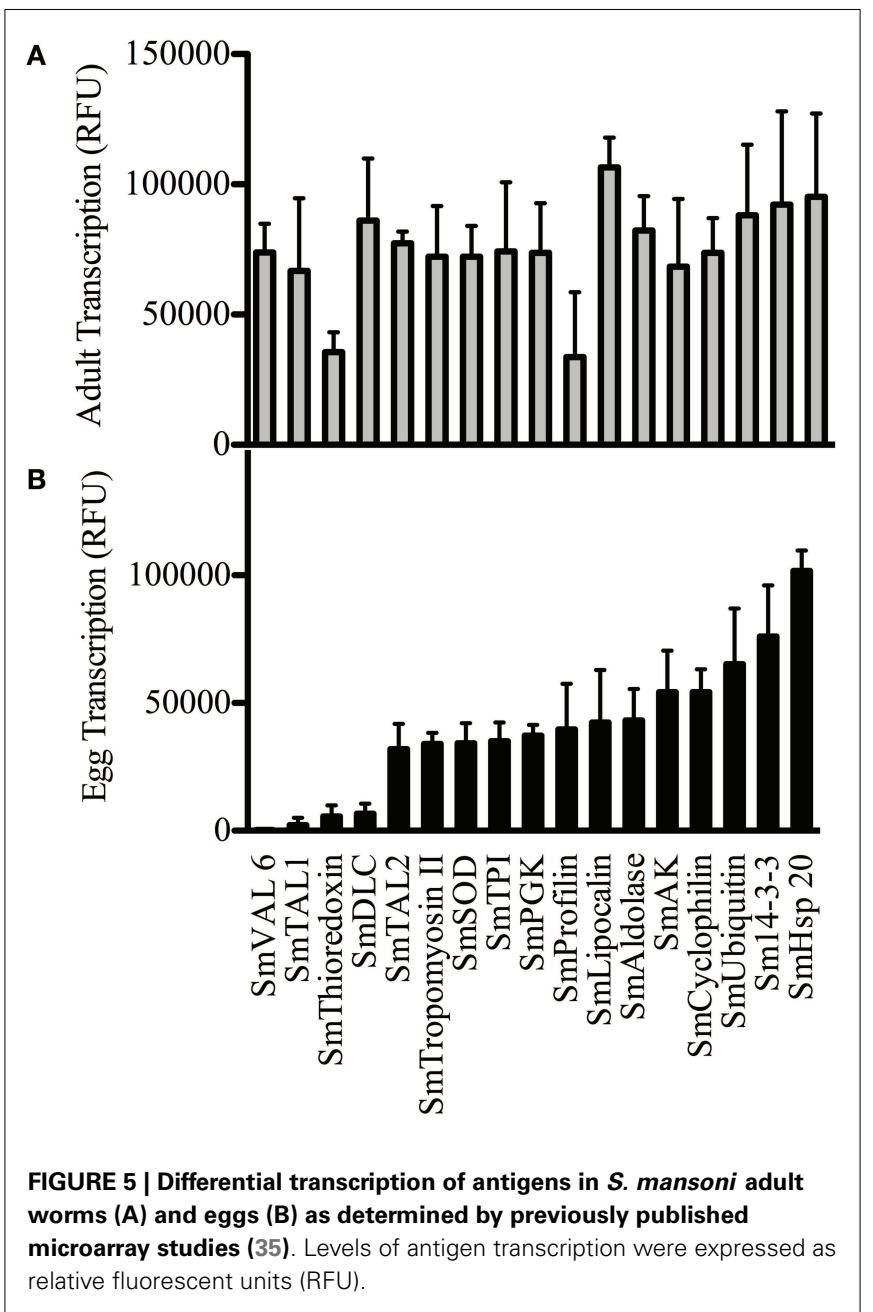

were targets for IgE, IgG4, and IgG1 in individuals infected with the parasite.

IgE responses were prevalent against all of the predicted parallergens, and against the known IgE target control antigens SmTAL1 and SmTropomyosin II. This study defines a novel set of antigens that are targeted by IgE in plasma from individuals with schistosomiasis mansoni, including SmProfilin, SmHSP20, SmPGK, SmSOD, SmCyclophilin, and SmThioredoxin. Several of these antigens are also the first examples of parallergens with similarity to known environmental allergens from the Profilin, HSP20 heat shock protein, $\mathrm{Cu} / \mathrm{Zn}$ Superoxide dismutase, and Thioredoxin allergen families, respectively. SmVAL-6 is a novel human IgE-binding parallergen in $S$. mansoni and along with previously identified hookworm Na-ASP-2 and SmVAL-4 parallergens is a further example of a parallergen with structural similarity to allergens from the CAP family $(18,39)$. IgE-binding to Cyclophilin from Echinococcus granulosus has also been previously reported in infected humans (40). IgE responses against SmTAL1 and SmTropomyosin II have been previously reported in S. mansoni endemic areas of Uganda, whilst responses against SmTPI and SmLipocalin were described in a recent study on infected indviduals from Brazil $(15,17,19)$. Despite being a predicted IgE targeted 

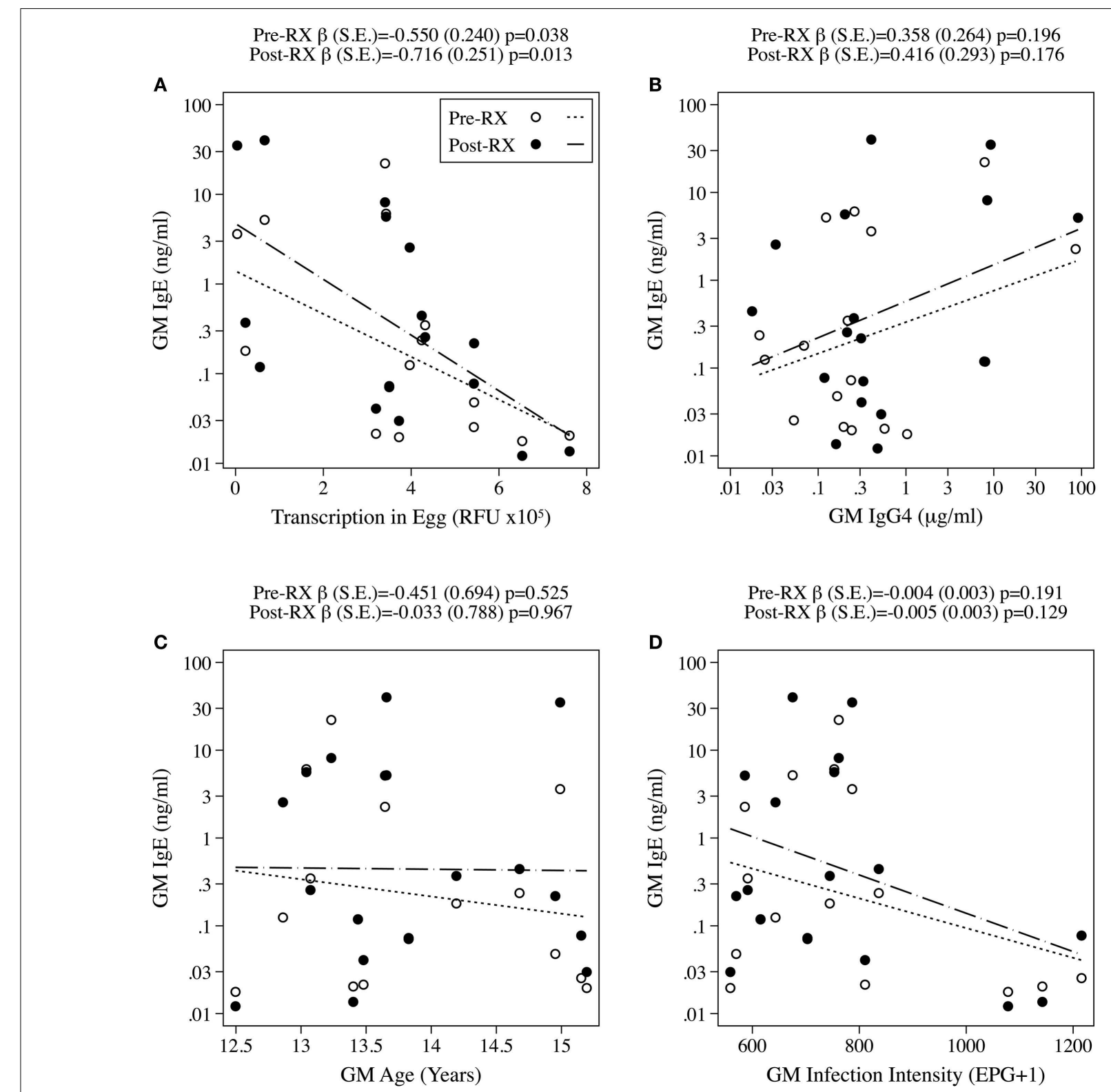

FIGURE 6 | Relationships between antigen-specific natural log normalized geometric mean (GM) IgE levels and transcription of antigen in egg (A)*, natural log normalized GM IgG4 levels (B), GM age (C), or GM infection intensity (D) in individuals that respond to either $\lg G 4$ or $\lg \mathrm{E}$

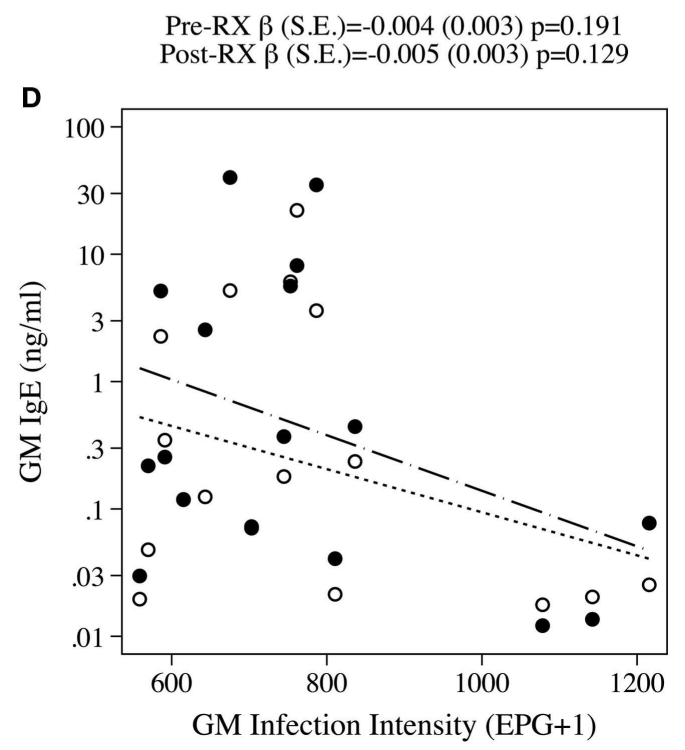

both pre- (open circles, short dashes) and post-treatment (filled circles, long dashes) with $\mathrm{PZQ}$ to a given antigen ( $\beta$, SE, and $\boldsymbol{p}$ value calculated by linear regression). ${ }^{*} \mathrm{SmHSP} 20$ was excluded from this plot as it exerted undue influence on the linear regression model (Cook's distance > 1).

antigen, SmTAL2 was not targeted by IgE. Other studies on human antibody responses against SmTAL2 in schistosomiasis mansoni endemic areas have shown that SmTAL2 may be targeted by IgE but only in early infection, which typically occurs in children under the age of 5, an age group not present in our study $(24,29)$.

Of the five $S$. mansoni antigens without allergen family counterparts, there was no strong evidence of IgE targeting of SmAK, SmUbiquitin, or Sm14-3-3; IgE responses were essentially absent from the cohort, being of both low prevalence and magnitude. SmDLC and SmAldolase however, were both targets of prevalent IgE responses. Currently, the Allfam dataset used to identify

IgE-binding proteins in this study only identifies class II aldolases (Pfam Acc. PF01116) as allergens, whilst SmAldolase is a class-I aldolase (Pfam Acc. PF00274) with distinctive structure associated with a separate Pfam domain (41). However, recent proteomic work has shown that a class-I Aldolase from maize (Zea mays) is targeted by IgE in maize allergic individuals, confirming SmAldolase as an allergen homolog $(42,43)$. Previous work on SmDLC had indicated that it was not the target of an IgE response (15), however that study used antigens that had been prepared under denaturing conditions, which may have affected their antigenicity. The evidence here that SmDLC is part of a novel class of 
IgE-binding proteins in S. mansoni, despite their absence from the allergen databases, may point to an important difference between allergens and some parallergens. In order to interact with the sufferer, allergens require the stability to survive the environment and cross mucosal barriers (44), whilst parallergens can be delivered directly into the blood and tissues by the parasite. Hence some classes of parasite IgE-binding proteins, such as DLC, may be absent from the allergy datasets as they are simply not seen by the immune systems of atopic individuals. Additionally, as illustrated for SmAldolase, the Allfam dataset, whilst comprehensive, is far from complete and it may be as more detailed proteomic studies of known allergen sources are completed further novel IgE-binding families are identified. For example, in a 2014 proteomic study on maize allergens, 6 of the 11 novel allergens found were not previously described in other allergenic sources and thus far are not present as Allfam families (42).

In the case of antibody responses to allergens in patients undergoing SIT and parasite IgE-binding proteins in chronic infections, IgG4 is thought to be induced as part of a protective anti-inflammatory regulatory response to IgE-mediated inflammation (reviewed (45) and (23), respectively). IgG4 responses were observed against all of the allergen-like antigens in this study in varying amounts. For SmHSP20, SmDLC, SmThioredoxin, SmTropomyosin II, SmAldolase, SmVAL-6, and SmTAL1, the majority of individuals that produced an $\operatorname{IgE}$ response against a given antigen also produced an IgG4 response against the same antigen providing further evidence for the role of IgG4 in a protective anti-inflammatory role in humans chronically infected with S. mansoni. Prevalent IgG4 responses were also observed against molecules with low prevalence IgE responses including SmTAL2 and SmUbiquitin. As discussed previously, it is thought that for antigens expressed strongly in eggs, such as SmTAL2, IgE antibodies are seen only during early infection (children $<5$ years) with IgE responses down regulated and replaced with IgG4 antibody responses in older individuals $(24,29)$. It is possible that this may also occur for SmUbiquitin, which is also expressed strongly in eggs, and that its absence from databases of known allergens is due to a similar process in which exposure to Ubiquitin, which is highly conserved in eukaryotes and abundant in other allergen sources such as birch pollen $(46,47)$, in atopic individuals results in down regulated IgE and up regulated IgG4.

Importantly, all of antigens tested were immunogenic, with differing proportions of infected individuals within the population producing an IgG1 response against each of the antigens. For several of these (SmPGK, SmSOD, SmLipocalin, SmTAL1, SmTAL2, SmTropomyosin II, Sm14-3-3, SmTPI, and SmDLC), responsiveness has been shown previously in infected humans and mice (15, 17, 48-52). However, novel human IgG1 responses were described to SmAK, SmUbiquitin, SmAldolase, SmHSP20, SmCyclophilin, SmThioredoxin, SmVAL-6, and SmProfilin. Crucially for Sm14-33, SmUbiquitin, and SmAK, to which IgE responses were low, a prevalent IgG1 response was found, confirming that the low-level of allergy-like responses was not due to poor immunogenicity.

Alignments of known parallergen and allergen structures including examples of Superoxide dismutase, Thioredoxin, and Lipocalin allergen families demonstrated that, despite relatively low sequence identity between matched parallergens and allergens, there was high gross structural similarity. This observation lends further weight to our hypothesis, which suggests that the structural similarity between allergens and metazoan parasite IgE-binding molecules defines the restriction of allergen families to a small proportion of structural domains. We predict that more extensive bioinformatic, crystallographic, and serological studies of whole parasite proteomes and the five remaining allergen super families (Prolamins, Subtilisin-like serine proteases, Bet v1-like proteins, Cupins, and Expansins) may reveal further parallergens in support of this hypothesis.

Studies on the SmTAL family of antigens suggest that sequestration of antigens may influence their overall levels of exposure to the immune system and consequently antibody levels (24). In schistosomiasis, the death of eggs trapped in tissue has been suggested to result in constant exposure of allergen-like molecules to the immune system, whilst pharmacological damage of adult worms results in exposure to sequestered antigens upon treatment with PZQ $(15,24)$. Consistent with this IgE, IgG4 and IgG1 antibody levels to both SmVAL-6 and SmDLC, antigens that are expressed in adult worms but not eggs, boosted significantly following treatment with PZQ. Conversely antigens expressed in both eggs and adults, including SmSOD, Sm14-3-3, and SmUbiquitin had significant decreases in specific antibody levels 5 weeks post-treatment with PZQ, which may be the result of decreased exposure to these antigens as eggs are no longer being produced. Previous investigations of serological responses in SIT have indicated that prolonged exposure to allergens may result in a decrease in serum IgE levels $(26,53)$. We found that for the majority of antigens in the study the relative level of expression of antigens in egg was negatively correlated with levels of IgE to that antigen. However, levels of SmHSP20 specific IgE were high despite being the molecule being strongly expressed in eggs. Intriguingly, the levels of IgG4 to SmHSP20 were between 30 and 35 times greater than next highest antigenspecific IgG4 levels (SmThioredoxin). Therefore we suggest that, for the majority of antigens, constant exposure to antigens due to the death of eggs results in a down-regulation of IgE against those antigens with the effective dose of antigen received (as indicated by transcription level in eggs) governing the level of IgE suppression. The data for SmHSP20 suggest that there may also be another mechanism for regulating deleterious IgE responses in which large quantities of IgG4 are produced in the absence of IgE down-regulation, the reasons for which require further investigation. The parallels between SIT and the natural immune response to chronic S. mansoni infection described here and elsewhere highlight the similarities between the immune responses to metazoan parasites and allergens and provide further evidence for the evolutionary origins of allergy-like responses in the immune reactions against metazoan parasites.

Our data, supporting the hypothesis that molecular structures associated with metazoan parasites determine the allergen landscape, have broad implications for the evolution and function of the $\operatorname{IgE}$ response consistent with its role of protection against these organisms. Endo- and ecto-macroparasites would have placed strong evolutionary pressure on the immune system, and specialized molecular mechanisms are likely to have developed to rapidly expand $\operatorname{IgE}$ responsiveness during parasite challenge and regulate harmful IgE-mediated immune responses. Whether 
operating through an antigen-specific mechanism, or invoking a new set of pathogen receptors, this hypothesis provides a novel, testable, explanation of allergy and allergens (54). In this setting, a more detailed analysis of functionally diverse $S$. mansoni antigens, guided by a structural bioinformatics pipeline based on known allergen structures, may provide a clearer picture as to the complex relationship between IgE production, regulatory IgG4 responses, and protective responses to the parasite and help elucidate the mechanisms of IgE targeting in both parasitic disease and allergy.

\section{ACKNOWLEDGMENTS}

We would like to thank the people of Namoni Village for their time and co-operation in the study as well as the field workers of the Ugandan Virus Research Institute and the Kenyan Medical Research Institute. Thanks go also to Shona Wilson, Joseph Mwatha, and Timothy Kamau, for their knowledge and skill in collecting and preparing samples for the study. We would also like to thank Maureen Laidlaw of the Schistosomiasis Research Group (University of Cambridge) for her expert technical assistance; Undergraduate students Aws Sadik, Matthew J Murray, Emma Robbins, and Emily Day for their hard work during final year projects in the Schistosomiasis Research Group; and Prof. Dame Janet Thornton at the European Bioinformatics Institute for her continued help and support. This work was supported by the Wellcome Trust via program WT $083931 / \mathrm{Z} / 07 / \mathrm{Z}$ and project WT 094317/Z/10/Z grants and by the European Commission via FP7-CP-IP-SICA scheme grant 242107.

\section{SUPPLEMENTARY MATERIAL}

The Supplementary Material for this article can be found online at http://www.frontiersin.org/Journal/10.3389/fimmu.2015.00026/ abstract

\section{REFERENCES}

1. Mari A, Scala E, Palazzo P, Ridolfi S, Zennaro D, Carabella G. Bioinformatics applied to allergy: allergen databases, from collecting sequence information to data integration. The Allergome platform as a model. Cell Immunol (2006) 244:97-100. doi:10.1016/j.cellimm.2007.02.012

2. Traidl-Hoffmann C, Jakob T, Behrendt H. Determinants of allergenicity. JAllergy Clin Immunol (2009) 123:558-66. doi:10.1016/j.jaci.2008.12.003

3. Radauer C, Bublin M, Wagner S, Mari A, Breiteneder H. Allergens are distributed into few protein families and possess a restricted number of biochemical functions. J Allergy Clin Immunol (2008) 121:847-52.e7. doi:10.1016/j.jaci.2008.01. 025

4. Palm NW, Rosenstein RK, Medzhitov R. Allergic host defences. Nature (2012) 484:465-72. doi:10.1038/nature11047

5. Fitzsimmons CM, Dunne DW. Survival of the fittest: allergology or parasitology? Trends Parasitol (2009) 25:447-51. doi:10.1016/j.pt.2009.07.004

6. Faulkner H, Turner J, Kamgno J, Pion SD, Boussinesq M, Bradley JE. Ageand infection intensity-dependent cytokine and antibody production in human trichuriasis: the importance of IgE. J Infect Dis (2002) 185:665-72. doi:10.1086/ 339005

7. Turner JD, Faulkner H, Kamgno J, Kennedy MW, Behnke J, Boussinesq M, et al. Allergen-specific IgE and IgG4 are markers of resistance and susceptibility in a human intestinal nematode infection. Microbes Infect (2005) 7:990-6. doi:10.1016/j.micinf.2005.03.036

8. Rihet P, Demeure CE, Bourgois A, Prata A, Dessein AJ. Evidence for an association between human resistance to Schistosoma mansoni and high anti-larval IgE levels. Eur J Immunol (1991) 21:2679-86. doi:10.1002/eji.1830211106

9. Fitzsimmons CM, Falcone FH, Dunne DW. Helminth allergens, parasite-specific ige, and its protective role in human immunity. Front Immunol (2014) 5:61. doi:10.3389/fimmu.2014.00061
10. Dunne DW, Butterworth AE, Fulford AJ, Ouma JH, Sturrock RF. Human IgE responses to Schistosoma mansoni and resistance to reinfection. Mem Inst Oswaldo Cruz (1992) 87(Suppl 4):99-103. doi:10.1590/S007402761992000800014

11. Hagan P, Blumenthal UJ, Dunn D, Simpson AJ, Wilkins HA. Human IgE, IgG4 and resistance to reinfection with Schistosoma haematobium. Nature (1991) 349:243-5. doi:10.1038/349243a0

12. Pritchard DI, Quinnell RJ, Walsh EA. Immunity in humans to Necator americanus: IgE, parasite weight and fecundity. Parasite Immunol (1995) 17:71-5. doi:10.1111/j.1365-3024.1995.tb00968.x

13. Matsuda H, Watanabe N, Kiso Y, Hirota S, Ushio H, Kannan Y, et al. Necessity of IgE antibodies and mast cells for manifestation of resistance against larval Haemaphysalis longicornis ticks in mice. J Immunol (1990) 144:259-62.

14. Jenkins JA, Breiteneder H, Mills ENC. Evolutionary distance from human homologs reflects allergenicity of animal food proteins. J Allergy Clin Immunol (2007) 120:1399-405. doi:10.1016/j.jaci.2007.08.019

15. Fitzsimmons CM, McBeath R, Joseph S, Jones FM, Walter K, Hoffmann KF, et al. Factors affecting human IgE and IgG responses to allergen-like Schistosoma mansoni antigens: molecular structure and patterns of in vivo exposure. Int Arch Allergy Immunol (2007) 142:40-50. doi:10.1159/000095997

16. Jenkins RE, Taylor MJ, Gilvary NJ, Bianco AE. Tropomyosin implicated in host protective responses to microfilariae in onchocerciasis. Proc Natl Acad Sci U S A (1998) 95:7550-5. doi:10.1073/pnas.95.13.7550

17. Silas S, Fitzsimmons CM, Jones FM, Pinot de Moira A, Wawrzyniak J, Tukahebwa EM, et al. Human IgE responses to different splice variants of Schistosoma man soni tropomyosin: associations with immunity. Int J Parasitol (2014) 44:381-90. doi:10.1016/j.ijpara.2014.02.004

18. Diemert DJ, Pinto AG, Freire J, Jariwala A, Santiago H, Hamilton RG, et al. Generalized urticaria induced by the Na-ASP-2 hookworm vaccine: implications for the development of vaccines against helminths. J Allergy Clin Immunol (2012) 130:169-76.e6. doi:10.1016/j.jaci.2012.04.027

19. Gaze S, Driguez P, Pearson MS, Mendes T, Doolan DL, Trieu A, et al. An immunomics approach to schistosome antigen discovery: antibody signatures of naturally resistant and chronically infected individuals from endemic areas. PLoS Pathog (2014) 10:e1004033. doi:10.1371/journal.ppat.1004033

20. Braschi S, Curwen RS, Ashton PD, Verjovski-Almeida S, Wilson A. The tegument surface membranes of the human blood parasite Schistosoma mansoni: a proteomic analysis after differential extraction. Proteomics (2006) 6:1471-82. doi:10.1002/pmic.200500368

21. Curwen RS, Ashton PD, Johnston DA, Wilson RA. The Schistosoma mansoni soluble proteome: a comparison across four life-cycle stages. Mol Biochem Parasitol (2004) 138:57-66. doi:10.1016/j.molbiopara.2004.06.016

22. Mutapi F, Bourke C, Harcus Y, Midzi N, Mduluza T, Turner CM, et al. Differential recognition patterns of Schistosoma haematobium adult worm antigens by the human antibodies IgA, IgE, IgG1 and IgG4. Parasite Immunol (2011) 33:181-92. doi:10.1111/j.1365-3024.2010.01270.x

23. Maizels RM, Yazdanbakhsh M. Immune regulation by helminth parasites: cellular and molecular mechanisms. Nat Rev Immunol (2003) 3:733-44. doi:10. $1038 /$ nril 183

24. Fitzsimmons CM, Jones FM, Stearn A, Chalmers IW, Hoffmann KF, Wawrzyniak J, et al. The Schistosoma mansoni tegumental-allergen-like (TAL) protein family: influence of developmental expression on human IgE responses. PLoS Negl Trop Dis (2012) 6:e1593. doi:10.1371/journal.pntd.0001593

25. Akdis CA, Akdis M. Mechanisms of allergen-specific immunotherapy. J Allergy Clin Immunol (2011) 127:18-27. doi:10.1016/j.jaci.2010.11.030

26. Jutel M, Jaeger L, Suck R, Meyer H, Fiebig H, Cromwell O. Allergen-specific immunotherapy with recombinant grass pollen allergens. JAllergy Clin Immunol (2005) 116:608-13. doi:10.1016/j.jaci.2005.06.004

27. Colley DG, Bustinduy AL, Secor WE, King CH. Human schistosomiasis. Lancet (2014) 383:2253-64. doi:10.1016/S0140-6736(13)61949-2

28. Wilson RA, Coulson PS. Immune effector mechanisms against schistosomiasis: looking for a chink in the parasite's armour. Trends Parasitol (2009) 25:423-31. doi:10.1016/j.pt.2009.05.011

29. Pinot de Moira A, Sousa-Figueiredo JC, Jones FM, Fitzsimmons CM, Betson M, Kabatereine NB, et al. Schistosoma mansoni infection in preschoolaged children: development of immunoglobulin E and immunoglobulin G4 responses to parasite allergen-like proteins. J Infect Dis (2013) 207:362-6. doi:10.1093/infdis/jis676 
30. Hoffmann KF, Fitzpatrick JM. Gene expression studies using self-fabricated parasite cDNA microarrays. Methods Mol Biol (2004) 270:219-36. doi:10.1385/159259-793-9:219

31. UniProt Consortium. Update on activities at the Universal Protein Resource (UniProt) in 2013. Nucleic Acids Res (2013) 41:D43-7. doi:10.1093/ nar/gks1068

32. Šali A, Blundell TL. Comparative protein modelling by satisfaction of spatial restraints. J Mol Biol (1993) 234:779-815. doi:10.1006/jmbi.1993.1626

33. Zinsser VL, Farnell E, Dunne DW, Timson DJ. Triose phosphate isomerase from the blood fluke Schistosoma mansoni: biochemical characterisation of a potential drug and vaccine target. FEBS Lett (2013) 587:3422-7. doi:10.1016/j.febslet. 2013.09.022

34. Cardoso RM, da Silva CH, de Araújo AP, Tanaka T, Tanaka M, Garratt RC. Expression and preliminary $\mathrm{X}$-ray diffraction studies of cytosolic $\mathrm{Cu}, \mathrm{Zn}$ superoxide dismutase from Schistosoma mansoni. Acta Crystallogr D Biol Crystallogr (2001) 57:1877-80. doi:10.1107/S0907444901014500

35. Fitzpatrick JM, Peak E, Perally S, Chalmers IW, Barrett J, Yoshino TP, et al. Antischistosomal intervention targets identified by lifecycle transcriptomic analyses. PLoS Negl Trop Dis (2009) 3:e543. doi:10.1371/journal.pntd.0000543

36. Pinot de Moira A, Fulford AJC, Kabatereine NB, Ouma JH, Booth M, Dunne DW. Analysis of complex patterns of human exposure and immunity to Schistosomiasis mansoni: the influence of age, sex, ethnicity and IgE. PLoS Negl Trop Dis (2010) 4(9):e820. doi:10.1371/journal.pntd.0000820

37. Pinot de Moira A, Jones FM, Wilson S, Tukahebwa E, Fitzsimmons CM, Mwatha $\mathrm{JK}$, et al. Effects of treatment on IgE responses against parasite allergen-like proteins and immunity to reinfection in childhood schistosome and hookworm coinfections. Infect Immun (2013) 81:23-32. doi:10.1128/IAI.00748-12

38. Fitzsimmons CM, Jones FM, Pinot de Moira A, Protasio AV, Khalife J, Dickinson HA, et al. Progressive cross-reactivity in IgE responses: an explanation for the slow development of human immunity to schistosomiasis? Infect Immun (2012) 80:4264-70. doi:10.1128/IAI.00641-12

39. Farias LP, Rodrigues D, Cunna V, Rofatto HK, Faquim-Mauro EL, Leite LCC. Schistosoma mansoni venom allergen like proteins present differential allergic responses in a murine model of airway inflammation. PLoS Negl Trop Dis (2012) 6:e1510. doi:10.1371/journal.pntd.0001510

40. Ortona E, Vaccari S, Margutti P, Delunardo F, Rigano R, Profumo E, et al. Immunological characterization of Echinococcus granulosus cyclophilin, an allergen reactive with IgE and IgG4 from patients with cystic echinococcosis. Clin Exp Immunol (2002) 128:124-30. doi:10.1046/j.1365-2249. 2002.01807.x

41. Ishiguro A, Homma M, Torii S, Tanaka K. Identification of Candida albicans antigens reactive with immunoglobulin $\mathrm{E}$ antibody of human sera. Infect Immun (1992) 60:1550-7.

42. Fonseca C, Planchon S, Pinheiro C, Renaut J, Ricardo CP, Oliveira MM, et al. Maize IgE binding proteins: each plant a different profile? Proteome Sci (2014) 12:17. doi:10.1186/1477-5956-12-17

43. Fonseca C, Planchon S, Renaut J, Oliveira MM, Batista R. Characterization of maize allergens - MON810 vs. its non-transgenic counterpart. J Proteomics (2012) 75:2027-37. doi:10.1016/j.jprot.2012.01.005

44. Chapman MD, Pomés A, Breiteneder H, Ferreira F. Nomenclature and structural biology of allergens. J Allergy Clin Immunol (2007) 119:414-20. doi:10.1016/j. jaci.2006.11.001

45. Fujita H, Soyka MB, Akdis M, Akdis CA. Mechanisms of allergen-specific immunotherapy. Clin Transl Allergy (2012) 2:2. doi:10.1186/2045-7022-2-2
46. Erler A, Hawranek T, Krückemeier L, Asam C, Egger M, Ferreira F, et al. Proteomic profiling of birch (Betula verrucosa) pollen extracts from different origins. Proteomics (2011) 11:1486-98. doi:10.1002/pmic.201000624

47. Goldstein G, Scheid M, Hammerling U, Schlesinger DH, Niall HD, Boyse EA. Isolation of a polypeptide that has lymphocyte-differentiating properties and is probably represented universally in living cells. Proc Natl Acad Sci U S A (1975) 72:11-5. doi:10.1073/pnas.72.1.11

48. Carvalho-Queiroz C, Cook R, Wang CC, Correa-Oliveira R, Bailey NA, Egilmez NK, et al. Cross-reactivity of Schistosoma mansoni cytosolic superoxide dismutase, a protective vaccine candidate, with host superoxide dismutase and identification of parasite-specific B epitopes. Infect Immun (2004) 72:2635-47. doi:10.1128/IAI.72.5.2635-2647.2004

49. Lee KW, Thakur A, Karim AM, Loverde PT. Immune response to Schistosoma mansoni phosphoglycerate kinase during natural and experimental infection: identification of a schistosome-specific B-cell epitope. Infect Immun (1995) 63:4307-11.

50. Brito CF, Fonseca CT, Goes AM, Azevedo V, Simpson AJ, Oliveira SC. Human IgG1 and IgG3 recognition of Schistosoma mansoni $14 \mathrm{kDa}$ fatty acid-binding recombinant protein. Parasite Immunol (2000) 22:41-8.

51. Schechtman D, Tarrab-Hazdai R, Arnon R. The 14-3-3 protein as a vaccine candidate against schistosomiasis. Parasite Immunol (2001) 23:213-7. doi:10.1046/j.1365-3024.2001.00378.x

52. Capron A, Capron M, Riveau G. Vaccine development against schistosomiasis from concepts to clinical trials. Br Med Bull (2002) 62:139-48. doi:10.1093/bmb/ 62.1 .139

53. Gleich GJ, Zimmermann EM, Henderson LL, Yunginger JW. Effect of immunotherapy on immunoglobulin $\mathrm{E}$ and immunoglobulin $\mathrm{G}$ antibodies to ragweed antigens: a six-year prospective study. J Allergy Clin Immunol (1982) 70:261-71. doi:10.1016/0091-6749(82)90062-8

54. Pontes-de-Carvalho L, Mengel JA. Question of nature: some antigens are bound to be allergens. Front Immunol (2014) 5:373. doi:10.3389/fimmu.2014.00373

Conflict of Interest Statement: The authors declare that the research was conducted in the absence of any commercial or financial relationships that could be construed as a potential conflict of interest. The Review Editor Adam Cunningham declares that, despite having collaborated on a publication in the last 2 years with author Rick M. Maizels, the review process was handled objectively.

Received: 19 September 2014; accepted: 12 January 2015; published online: 03 February 2015.

Citation: Farnell EJ, Tyagi N, Ryan S, Chalmers IW, Pinot de Moira A, Jones FM, Wawrzyniak J, Fitzsimmons CM, Tukahebwa EM, Furnham N, Maizels RM and Dunne DW (2015) Known allergen structures predict Schistosoma mansoni IgE-binding antigens in human infection. Front. Immunol. 6:26. doi: 10.3389/fimmu.2015.00026

This article was submitted to Microbial Immunology, a section of the journal Frontiers in Immunology.

Copyright (c) 2015 Farnell, Tyagi, Ryan, Chalmers, Pinot de Moira, Jones, Wawrzyniak, Fitzsimmons, Tukahebwa, Furnham, Maizels and Dunne. This is an open-access article distributed under the terms of the Creative Commons Attribution License (CC $B Y)$. The use, distribution or reproduction in other forums is permitted, provided the original author(s) or licensor are credited and that the original publication in this journal is cited, in accordance with accepted academic practice. No use, distribution or reproduction is permitted which does not comply with these terms. 